\title{
A laser-based eye-tracking system
}

\author{
KENJI IRIE, BRUCE A. WILSON, and RICHARD D. JONES \\ University of Canterbury, Christchurch, New Zealand \\ and Christchurch Hospital, Christchurch, New Zealand \\ PHILIP J. BONES \\ University of Canterbury, Christchurch, New Zealand \\ and \\ TIM J. ANDERSON \\ Christchurch Hospital, Christchurch, New Zealand
}

\begin{abstract}
This paper reports on the development of a new eye-tracking system for noninvasive recording of eye movements. The eye tracker uses a flying-spot laser to selectivelyimage landmarks on the eye and, subsequently, measure horizontal, vertical, and torsional eye movements. Considerable work was required to overcome the adverse effects of specular reflection of the flying-spot from the surface of the eye onto the sensing elements of the eye tracker. These effects have been largely overcome, and the eye-tracker has been used to document eye movement abnormalities, such as abnormal torsional pulsion of saccades, in the clinical setting.
\end{abstract}

Quantitative eye movement measurements can provide invaluable information for the diagnosis and study of various neurological disorders and are an essential tool in fundamental research on the oculomotor system (Leigh \& Zee, 1999).

Human eye movements can be broken down into three distinct axes of rotation: horizontal, vertical, and torsional. Although horizontal and vertical eye movements are true rotations around the center of the eye, these can be usefully described as translational, albeit with units of angular measure. The primary function of these movements is to bring and keep the center of images of interest on the eye's fovea. Eye movements can have velocities up to $600 \% \mathrm{sec}$ (Leigh \& Zee, 1999) and power spectral components up to $50 \mathrm{~Hz}$ (spectral analysis of own data). Eye movements often contain both translational and torsional components. Some torsional abnormalities, such as pathological torsion pulsion (Anderson \& MacAskill, 1998), may be present only during translational eye movement.

A number of methods have been proposed or used for measuring eye movements, each with different features and levels of performance. Of particular importance is whether eye movements need to be measured only in one dimension (e.g., horizontal), in two dimensions (horizontal and vertical), or in three dimensions (translational plus torsional). The target goal of our laser-based eye tracker is

The laser-based eye tracker described in this paper is not available as a commercial product, but consideration is being given to pursuing this possibility pending the outcome of further development work. K.I., R.D.J., P.J.B., and T.J.A. are members of the Christchurch Movement Disorders and Brain Research Group. Correspondence concerning this article should be addressed to K. Irie, Lincoln Technology, P. O. Box 133, Lincoln, Christchurch 8152, New Zealand (e-mail: iriek@ lincoln. ac.nz). to track eye movements in three dimensions. A summary of the most common methods for measuring three-dimensional (3-D) eye movements follows.

Several methods are based on tracking contact lenses. The performance of these systems is typically very good, but all are invasive, uncomfortable, and often require a topical anaesthetic. Matin and Pearce (1964) developed a scleral contact lens system that uses a pair of noncoplanar 4-mm-diameter mirrors embedded in the surface of the lens on opposite sides of the pupil. Their system has a resolution of $0.00028^{\circ}$ within a range of $\pm 10^{\circ}$ for all three dimensions and has a flat frequency response up to $1.3 \mathrm{kHz}$. Robinson (1963) used a pair of magnetic search coils placed around the head to induce an electrical current into a pair of orthogonal magnetic coils placed in a scleral contact lens. Translational eye movements could be measured down to $0.25^{\circ}$ over a $\pm 20^{\circ}$ range and torsional movements could be resolved down to $0.0042^{\circ}$. Their system had a bandwidth of $1.0 \mathrm{kHz}$.

Methods based on tracking the retina (Young \& Sheena, 1975) and the fundus (Kawai, Tamura, Kani, \& Kariya, 1986) have been developed that can achieve bandwidths up to $60 \mathrm{~Hz}$ (Mulligan, 1997), although most fail to exceed $5 \mathrm{~Hz}$. Photographic images have also been used to measure eye movements, but the long postprocessing time required to develop and analyze the photographs severely limits this approach.

In the last 5 years or so, video-based systems have taken on a prominence in the eye-tracking world. The advent of digital recording devices (CCD and CMOS cameras) has led to video-based systems' becoming smaller, more reliable, and substantially faster than earlier analogue recording devices. An example is Alphabio's “Eyeputer" (http://www. electronica.fr/alphabio), which can sample at up to $480 \mathrm{~Hz}$ 
with a horizontal range of $\pm 30^{\circ}$, vertical range of $\pm 20^{\circ}$, and a torsional range of $\pm 45^{\circ}$. It has a precision of $\pm 0.3^{\circ}$ translationally and $\pm 0.2^{\circ}$ torsionally at this sample rate, although the horizontal and vertical precision can be improved to $\pm 0.1^{\circ}$ by reducing the sample rate to $60 \mathrm{~Hz}$. Other manufacturers of camera-based systems include Applied Science Laboratories (http://www. a-s-1.com), SKALAR Medical BV (http://www.wirehub. nl/ skalar), SensorMotoric Instruments (http://www. smi.de), and Chronos Vision GmbH (Clarke, Ditterich, Drüen, Schönfeld, \& Steineke, 2002).

Prior to the development of high frame rate video-based eye trackers, it was not possible to measure high-bandwidth saccadic eye movements in three dimensions by noninvasive measures. Consequently, in 1994, we commenced development of a high-speed 3-D laser-based flying-spot eye-tracking system (Jones, Wilson, \& Bones, 1996; Wilson, Anderson, Preddie, Jones, \& Bones, 1996a, 1996b; Wilson, Jones, \& Bones, 1995). By partially imaging the eye with a low-power eye-safe laser, it was recognized that it would be possible to achieve high-speed, high-bandwidth noninvasive 3-D eye tracking with only a fraction of the data processing that would be required for processing full video images of the eye. The spatial resolution is limited only by the laser spot size and the sampling frequency of the sensing system. The resolution in time is limited only by the speed of the sensors detecting the backscattered light, the bandwidth of the mechanical deflection apparatus, and the real-time computing capacity. This paper describes our laser-based eye-tracking system and, in particular, outlines some of the major obstacles met along the way and how they have been overcome to achieve a system with a performance suitable for both clinical and research applications. As far as the authors are aware, this is the first eye tracker to use a laser for tracking 3-D eye movements.

\section{SYSTEM DESCRIPTION}

\section{System Overview}

The eye tracker is essentially a specialized flying-spot scanner and consists of four main subsystems.

1. A 650-nm wavelength, 1-mW red laser (attenuated to $0.15 \mathrm{~mW}$ ) focused to produce an illumination spot of $53 \mu \mathrm{m}$ on the eye (complying with Australian Standard AS2211 [1991] for laser safety; Eckert, 1996).

2. Mirrors that steer the small spot of illumination across the eye. The beam-steering system consists of two galvanometer-driven beam-steering mirrors, servo controllers, and a digital-to-analogue converter. The mirrors are located above the subject's eye.

3. A receiver that measures the reflected light from the eye, consisting of two sampling channels, each with two photodiode sensors. The signals from the photodiodes are passed through a transimpedance amplifier and a variablegain amplifier before being digitized by an analogue-todigital converter card on a PC running MS-DOS.

4. A computer that controls the scanner, stores and processes data, and interacts with the user. The eye tracker software tracks the pupil in real time and directs the beamsteering mirrors to keep a laser scan pattern centered on the pupil. The system currently operates at 169 scans per second. The torsional movements are too computationally expensive to perform in real time on the current hardware and are calculated off line.

A block diagram of the eye tracker is shown in Figure 1. The headset of the eye tracker can be seen modeled in Figure 2.

Scan cycle. The scan cycle is a series of sweeps to partially image the eye so that the eye's translational and torsional position can be determined. The eye tracker determines the position of the eye on the basis of a horizontal sweep, a vertical sweep, and a circular sweep. In addition

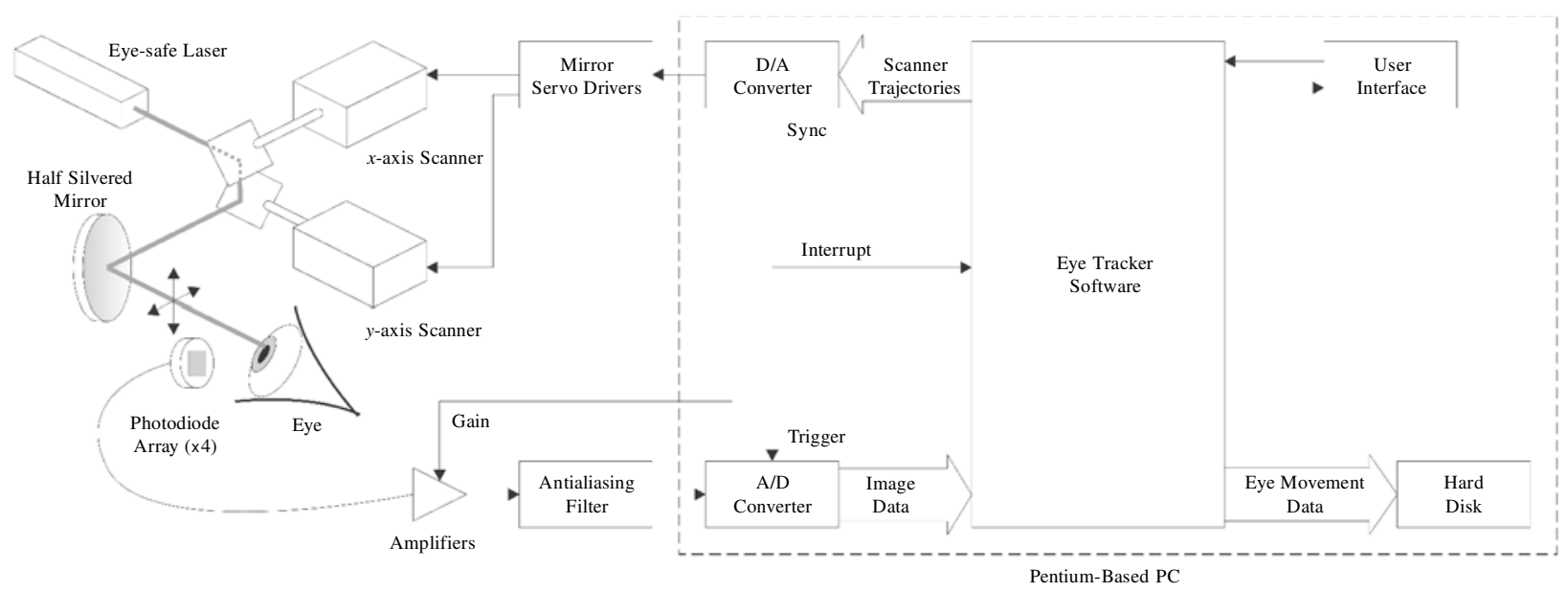

Figure 1. Block diagram of the eye tracker system. 


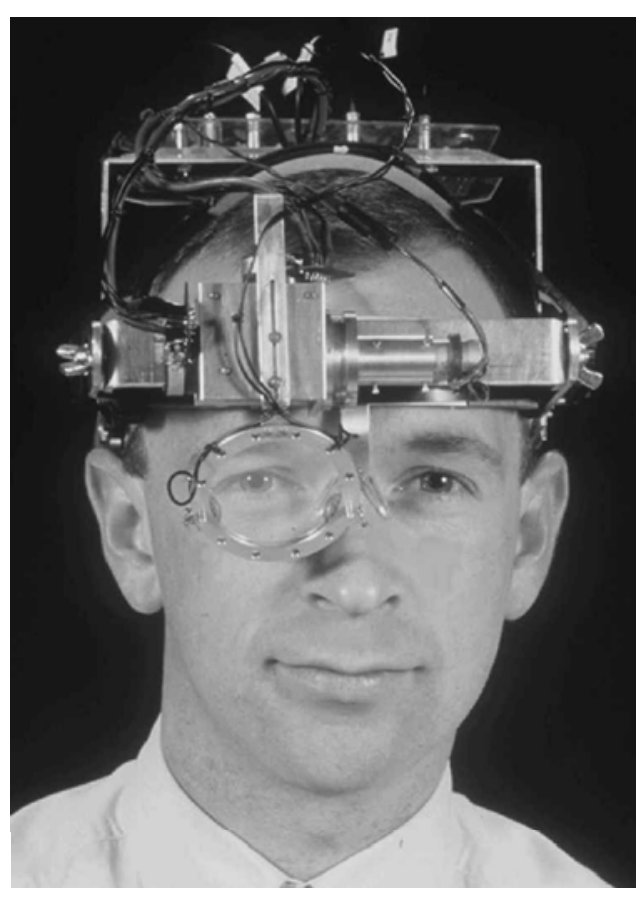

Figure 2. Front view of the eye tracker headset.

to these three sweeps, there is a fly-back period between sweeps to position the spot ready for the next scan. There are, therefore, six operations, or phases, that make up each cycle of measurement. Each sweep over the iris and pupil provides an image of the reflectivity of the eye along a line. Figure 3 shows the trajectory of the laser spot through these six phases of the scan cycle. Phase 1 moves the beam to the start point of the vertical sweep, whereas Phase 2 is the vertical sweep across the iris and pupil. From this ver- tical sweep, the edges of the pupil are found, and thus, the vertical center of the pupil can be located. The setup for the circular sweep is performed in Phase 3 , and Phase 4 records the iris pattern over a $270^{\circ}$ arc. Torsion of the eye is determined by cross-correlation of consecutive circular sweeps. Phase 5 moves the beam to the start point for the horizontal sweep, whereas Phase 6 is the horizontal sweep across the iris and pupil. From this horizontal sweep, the edges of the pupil are found, and thus, the horizontal center of the pupil can be located.

The size of the pupil can change over the course of the measurement period, but abnormal pupillary shape (e.g., ovoid) may result in small inaccuracies in estimation of translational and torsional positions. Rare defects of the iris, such as aniridia (congenital absence of iris), preclude measurement of torsional movements.

At the start of an eye movement recording session, the scan pattern is placed approximately over the subject's pupil/iris. The eye tracker is then allowed to "lock on" to the subject's pupil (and a position calibration performed) before eye movements are recorded. If the edges of the pupil are not detected in both the horizontal and the vertical sweeps over several consecutive scans, the eye tracker enters a search mode and actively seeks the pupil until it "locks on" to the subject's pupil again.

Data processing. The eye tracker software controls the collection, processing, and storage of scan information. The eye tracker software tracks the pupil in real time and directs the beam-steering mirrors to keep the scan pattern centered on the pupil. A typical example of a horizontal (or a vertical) sweep of the eye is shown in Figure 4. The pupil is indicated by the low-level signal area in the center of the sweep. The iris is seen as the higher level signal either side of the pupil region, and the edges of the pupil can be seen as the sharp signal steps in between the iris and the pupil. The center position of the pupil can be de-

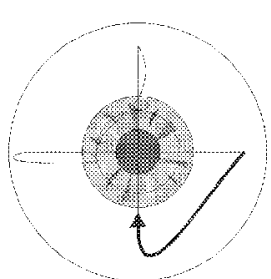

Phase 1

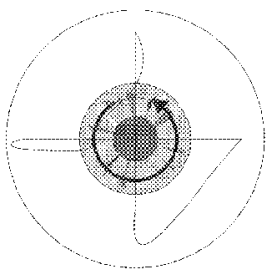

Phase 4

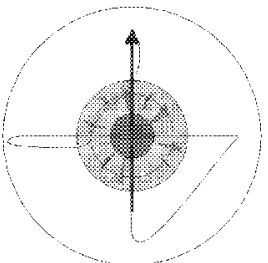

Phase 2

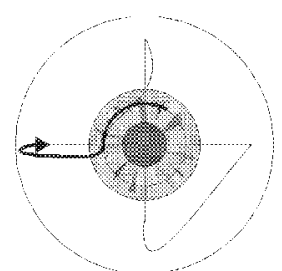

Phase 5

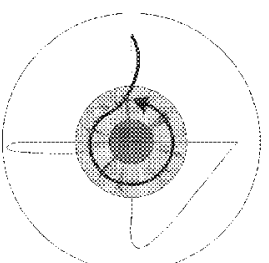

Phase 3

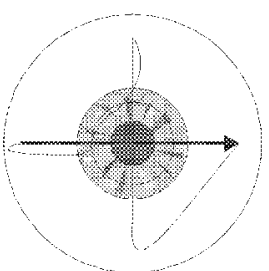

Phase 6

Figure 3. The six phases of a scan cycle. 


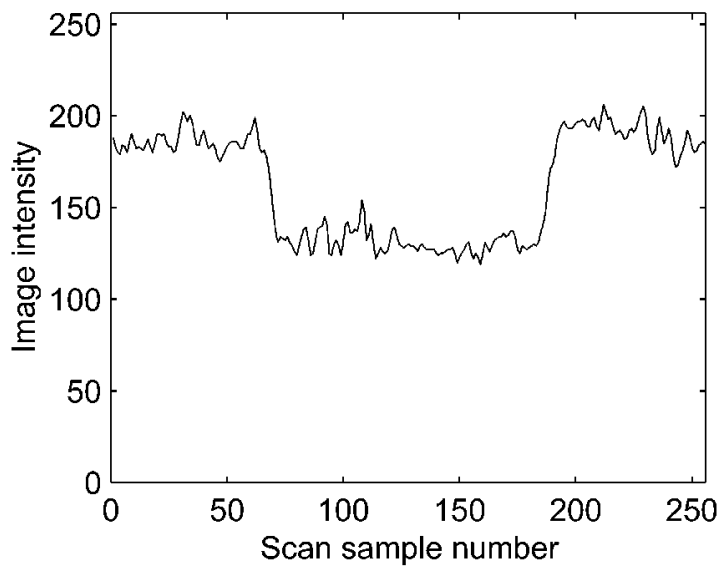

Figure 4. A typical horizontal sweep recording from the eye tracker.

termined from the top, bottom, left, and right edges of the horizontal and vertical sweeps.

The entire torsion estimation process is based on imaging features on the iris. The features are primarily composed of radial striations. By recording images of the iris, torsional eye movements can be estimated by correlating subsequent images with each other. Although this is a relatively straightforward process, it is too computationally expensive to be undertaken in real time on the current hardware. Instead, the scans are written to hard disk, and the circular sweeps are correlated off line to estimate torsional eye movements.

\section{OBSTACLES}

\section{Specular Reflections on Translational Tracking}

Paramount in achieving a clinically useful performance from the eye tracker is achieving reliable and accurate tracking of the pupil. Both translational and torsional measurements are affected by the quality of the pupil tracking. Initially, the eye tracker performed erratically, owing to its being unable to reliably track translational eye movements. Specular reflections were determined to be a major cause of tracking error as a result of the laser beam's reasonably frequently reflecting directly off the eye surface onto one or more of the four sensors. Figure 5 illustrates the specular reflection process. The sensors had been chosen and positioned so as to best detect the backscattered light from the eye but became saturated when the much higher intensity specular reflection passed onto them. Two levels of specular reflection were identified: saturating specular reflection, which saturated the photodiode output from an entire sweep and occurred when the laser beam reflected onto one of the photodiodes for 1-2 msec, and nonsaturating specular reflection, which affected only the part of the sweep that the specular reflection occurred upon. An example of saturating specular reflections upon a horizontal sweep is shown in Figure 6. Figure 6A has a strong specular reflection occurring over the left boundary between the pupil and the iris. This biases the pupil edge detection algorithm, which will inaccurately determine the pupil position. Figure $6 \mathrm{~B}$ has two strong specular reflections apparent through the horizontal sweep, occurring near the edges between the pupil and the iris. This also biases the pupiledge-detection algorithm. The result of these strong specular reflections is gross oscillation or loss of pupil tracking.

A two-part solution was implemented to reduce the erroneous eye tracking caused by specular reflection. First, the four sensors were placed around the eye to utilize the dual-channel sampling capability of the eye tracker system so as to ensure that at least one sampling channel is free from specular reflections at all times. With the sensors positioned around the eye as shown in Figure 7, a horizontal sweep can saturate only one of the sensor pairs at
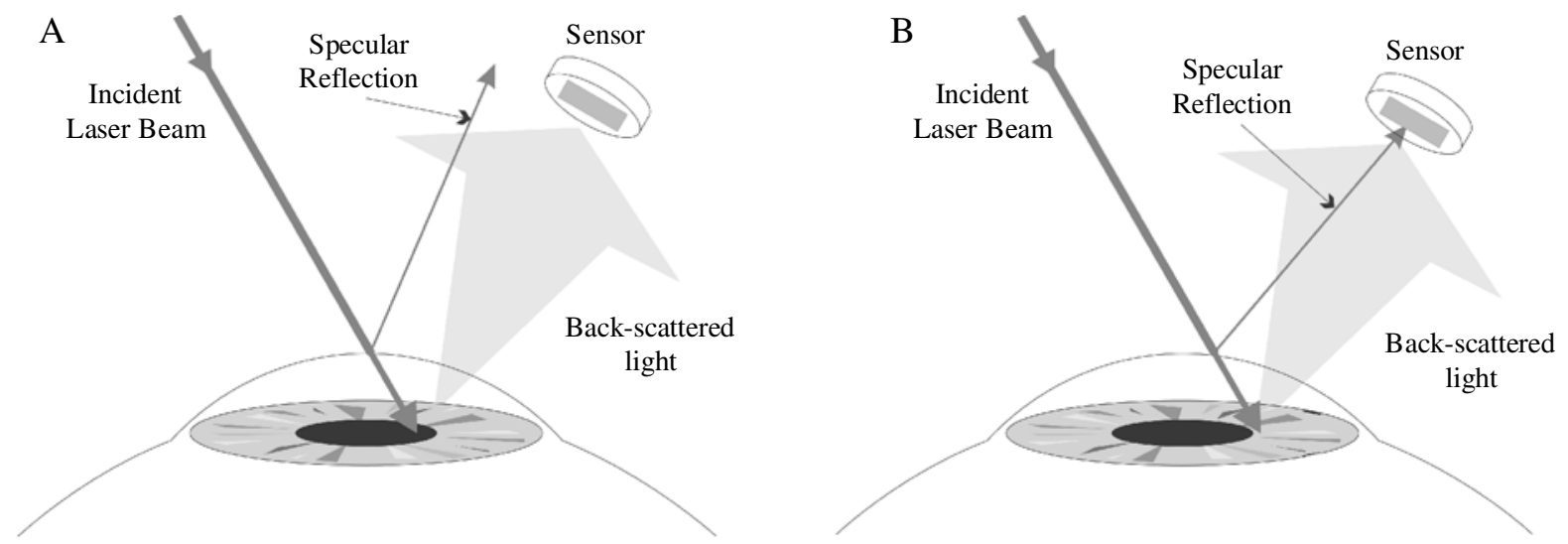

Figure 5. Diagram demonstrating (A) how specular reflection occurs and (B) how certain angles of incidence cause specular reflection to directly impose on a sensor. 

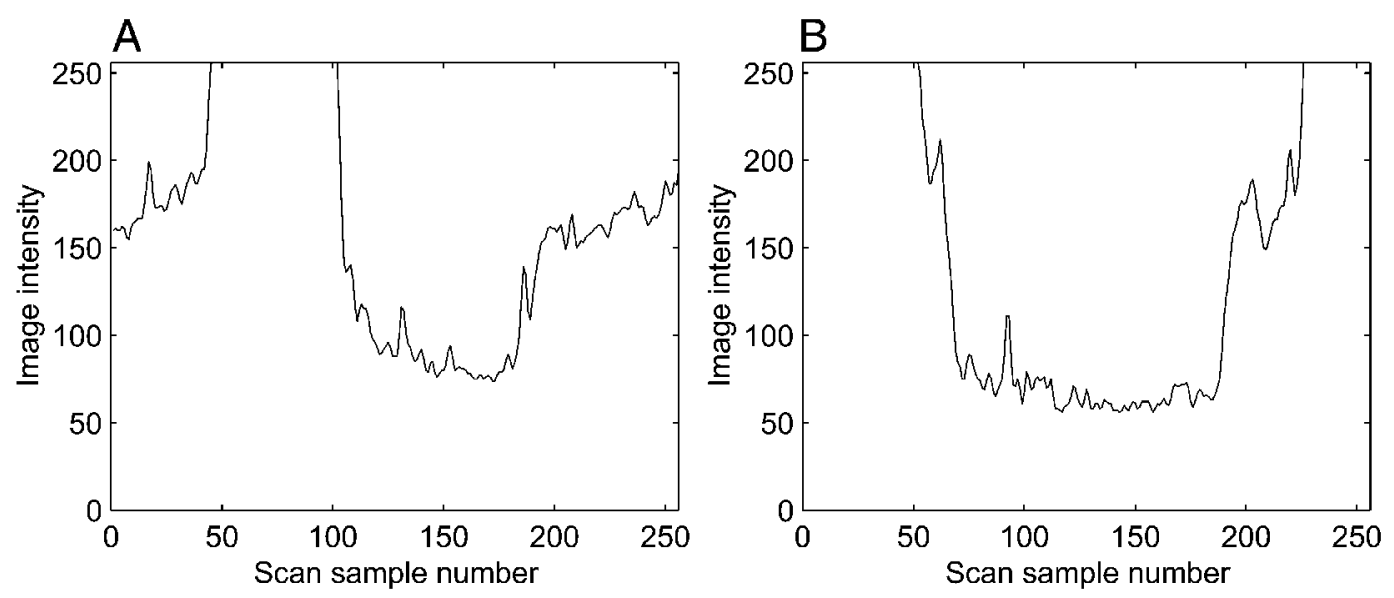

Figure 6. Horizontal sweep recordings from the eye tracker on the translational eye movement simulator showing saturating specular reflections: (A) a specular reflection occurring during a scan of the pupil and (B) two specular reflections, one on the pupil/iris boundary and one on the iris.

any given time, since the projected specular reflection is a slightly curved horizontal line. Similarly, during a vertical sweep, only one sensor (and, therefore, one sampling channel) can be saturated at any given time, since the projected specular reflection is a slightly curved vertical line.

The second part of the solution was to minimize the effect of any remaining specular reflections. By utilizing the dual-channel sampling capability of the eye tracker, many of the unwanted effects of saturating specular reflections were overcome by developing an algorithm capable of detecting and removing data contaminated by saturating specular reflections.

Figure 8 shows the major components of the sampling and channel selection subsystem of the eye tracker implemented to minimize the influence of any remaining saturating specular reflections. The optics have been carefully positioned so as to ensure that at least one of the sampling channels is free from specular reflection during any sweep. Data from the sensor pairs are summed in hardware on the eye tracker headset and are sampled by the A/ D converter board in the eye tracker PC. The channel selection algorithm is passed data from the two sampling channels. The two data streams (each 8-bit resolution) are checked for any saturation (i.e., signal levels of 255), and there are four possible resulting cases: Both channels are free of saturations; Channel 1 contains saturations, whereas Channel 2 does not; Channel 2 contains saturations, whereas Channel 1 does not; and both channels contain saturations. To determine the position of the center of the pupil, the algorithm selects the channel with no saturation and the widest pupil width detected.

\section{Distortions Affecting Torsional Tracking}

The flying-spot approach to measuring eye movements inherently brings several types of distortion into the torsional image. All image-based systems are susceptible to specular reflections from the eye that may render part of the torsional image unusable. A scanning eye position tracking system (as opposed to an absolute eye position measurement system, such as some contact lens systems and the video-based systems) suffers from additional time delays in the tracking feedback. These time delays can distort the recorded data and reduce the accuracy and reliability of the torsional eye tracking. Geometric distortions can also occur because of the spherical imaging of the surface of the eye. Each of these distortions, although independent of each other, may occur at the same time. Also, inherent in the eye tracker sampling process is an intensity gradient (brightness variation) along the recorded image that is dependent on the sensor positions relative to the scan pattern position. This implies that not only do the effects of the distortions need to be reduced, but also the recorded data need to be filtered to remove the intensity gradients, which can severely bias the cross-correlation algorithm.

The most destructive distortion by far is the specular reflections. Specular reflections are additive, and image data corrupted by saturating specular reflections from the sur-

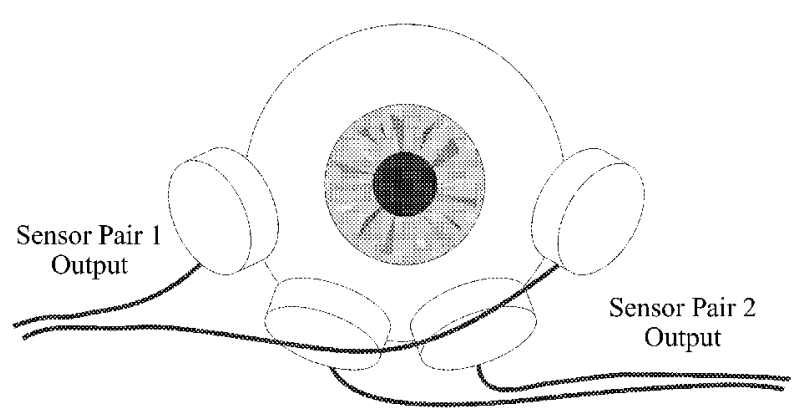

Figure 7. A set of sensor positions with a good balance between specular reflection minimization and signal levels. The sensors are placed 1-2 cm in front of the subject's eye. 


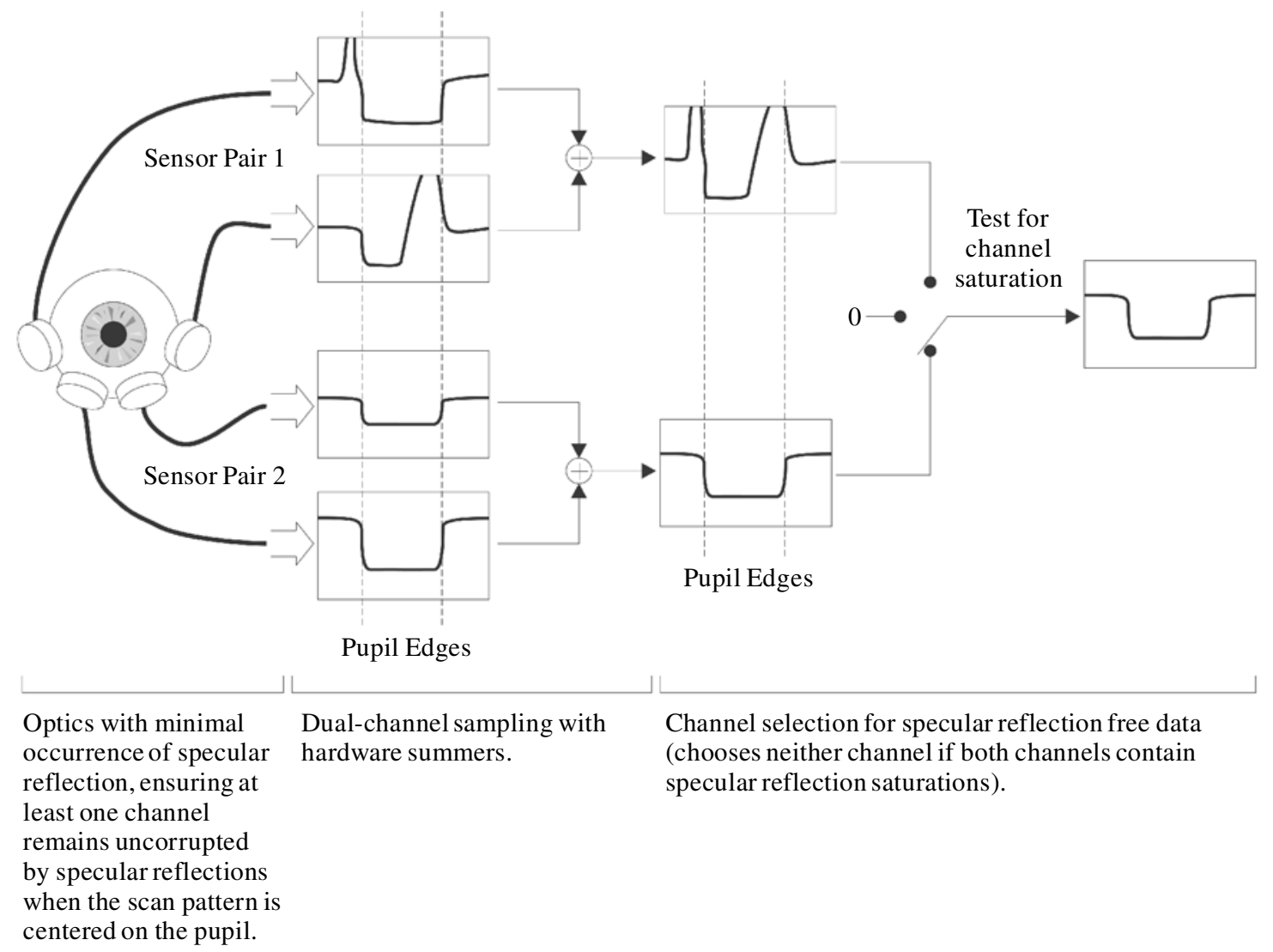

Figure 8. The dual-channel sampling and channel selection eye tracker subsystem.

face of the eye cannot be recovered. However, they severely bias the cross-correlation algorithm and need to be removed. Shown below in Figure 10A is a torsional recording saturated by specular reflection (further specular reflection in a torsional recording can be seen below in Figure 12A). As the cross-correlation function multiplies the two signals to be correlated, the removal of the effect of the saturating specular reflection can be achieved by setting areas of saturating specular reflection to zero. This is achieved by multiplying the original signal with a mask signal consisting of $1 \mathrm{~s}$ where nonsaturated data are present and 0 s where saturation occurs. Saturations are detected as signals equal to the limit of the 8-bit A/D output (integer value, 255). The setting of values within signals to 0 can bias the cross-correlation function. The result of the crosscorrelation needs careful unbiasing to ensure that the removal of saturating specular reflections does not dominate the cross-correlation.

In order to better illustrate this, a series of crosscorrelations is shown in Figure 9. Figure 9A shows the cross-correlation of series of two rectangular signals. The result of the cross-correlation is a triangular shaped signal. This represents the bias inherent in noncircular correlations. The result from the cross-correlation should be a horizontal line with no peak, signifying that the two input rectangular signals are equal across their entirety. The right-hand diagram of Figure 9A is the triangular shaped cross-correlation result divided by itself. Dividing the results of cross-correlations by this reference weighting signal unbiases the cross-correlation.

Figure 9B shows the same cross-correlation process as in Figure 9, but with two strong features added to the original input signals. The result of the cross-correlation is a slightly wavy triangular shape. The result is then divided by the reference weighting signal, and the cross-correlation shown on the right of Figure 9B. The peak of the result is in the very center of the signal, showing that the two input signals have the highest correlation when they coincide.

An example of the problem of setting values within the input signals to 0 is shown in Figure 9C. Ten samples on the right-hand side of one of the input signals have been arbitrarily set to 0 to simulate the removal of a saturating specular reflection. The resulting biased and unbiased cross-correlations are distorted, and the peak that should be located in the center of unbiased cross-correlation is reduced and no longer the maximum point of the crosscorrelation.

The cause of the distortion is due to the 0-values introduced into the signal. One solution that would improve the result of the cross-correlation is to change the reference weighting signal to incorporate the effect of adding 0 values to the signals. The reference weighting is formed 
A
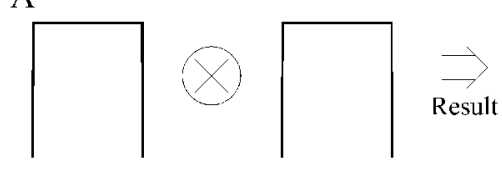

B
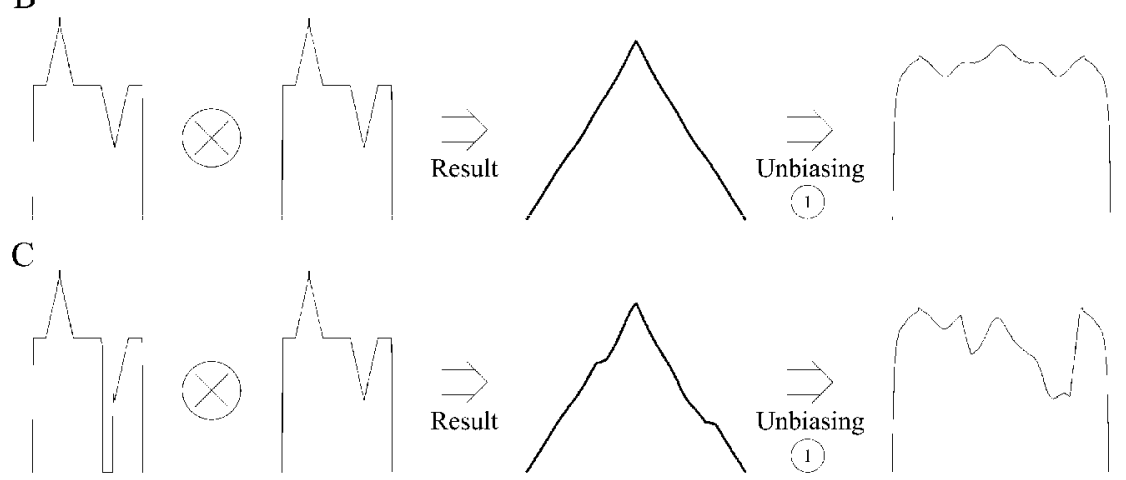

$\mathrm{D}$
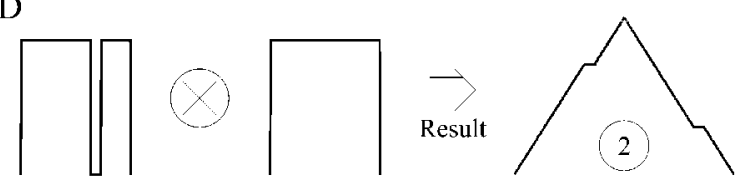

Weighting 2

$\mathrm{E}$
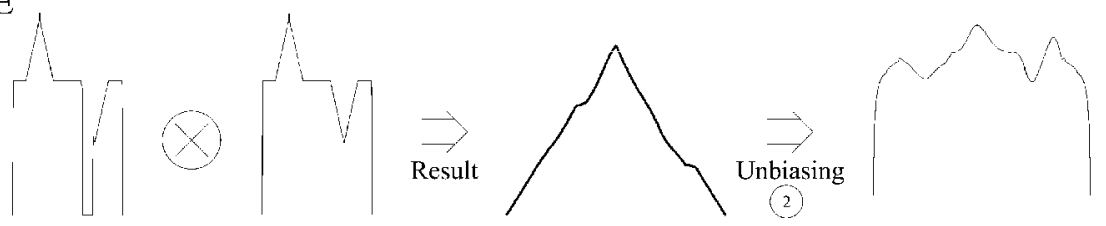

Figure 9. A series of diagrams illustrating the use of weightings to unbias noncircular cross-correlation results. (A) generation of a reference weighting that can be used to unbias other noncircular cross-correlations of the same length and same overall signal level. (B) Two signals that when unbiased by the reference weighting shown in panel $A$ have a peak in the center of the cross-correlation result, meaning that the signals are best correlated when there is no offset between the two signals. (C) The addition of 0 -values within one of the input signals to simulate specular reflection removal alters the cross-correlation result unbiased with the reference weighting shown in panel $A$. (D) The generation of a new weighting to unbias the cross-correlation shown in panel $C$. (E) The cross-correlation of the signals shown in panel $C$ with the new weighting signal unbiasing the result. The peak of the unbiased signal is now back in the center of the result, meaning that the signals are best correlated when there is no offset between them.

by the cross-correlation of two signals sampled at Level 1, each being the same length as the input signals. A modification to the input signals of the reference weighting cross-correlation can be made to incorporate the areas of the signals being unbiased that have been set to 0 . Figure 9D illustrates the production of a new weighting signal, with one of the input signals having 0 -values in the same place as the signal being unbiased. The resulting weighting signal is triangular in shape with ridges. When the result of the biased cross-correlation in Figure $9 \mathrm{C}$ is divided by the new weighting signal, the result is a much more pronounced center peak, as shown in Figure 9E, which is once again the maximum point of the crosscorrelation.
The process of removing saturating specular reflections gives a substantial improvement in the torsional results. Without this process, torsional estimation would tend to follow the paths of specular reflection that are almost always seen in torsional images

Intensity gradients in torsional signals can bias the cross-correlation process and produce erroneous results. The gradients occur because the sensor coverage of the eye is uneven, meaning that average light levels throughout the recorded data can vary. However, the gradients are low frequency in nature and can be removed with a highpass filter (a 32-tap Hamming filter with a normalized cutoff frequency of $0.15 f_{\mathrm{S}}$, where $f_{\mathrm{S}}$ is the sampling frequency, was used). Its performance was, however, severely 

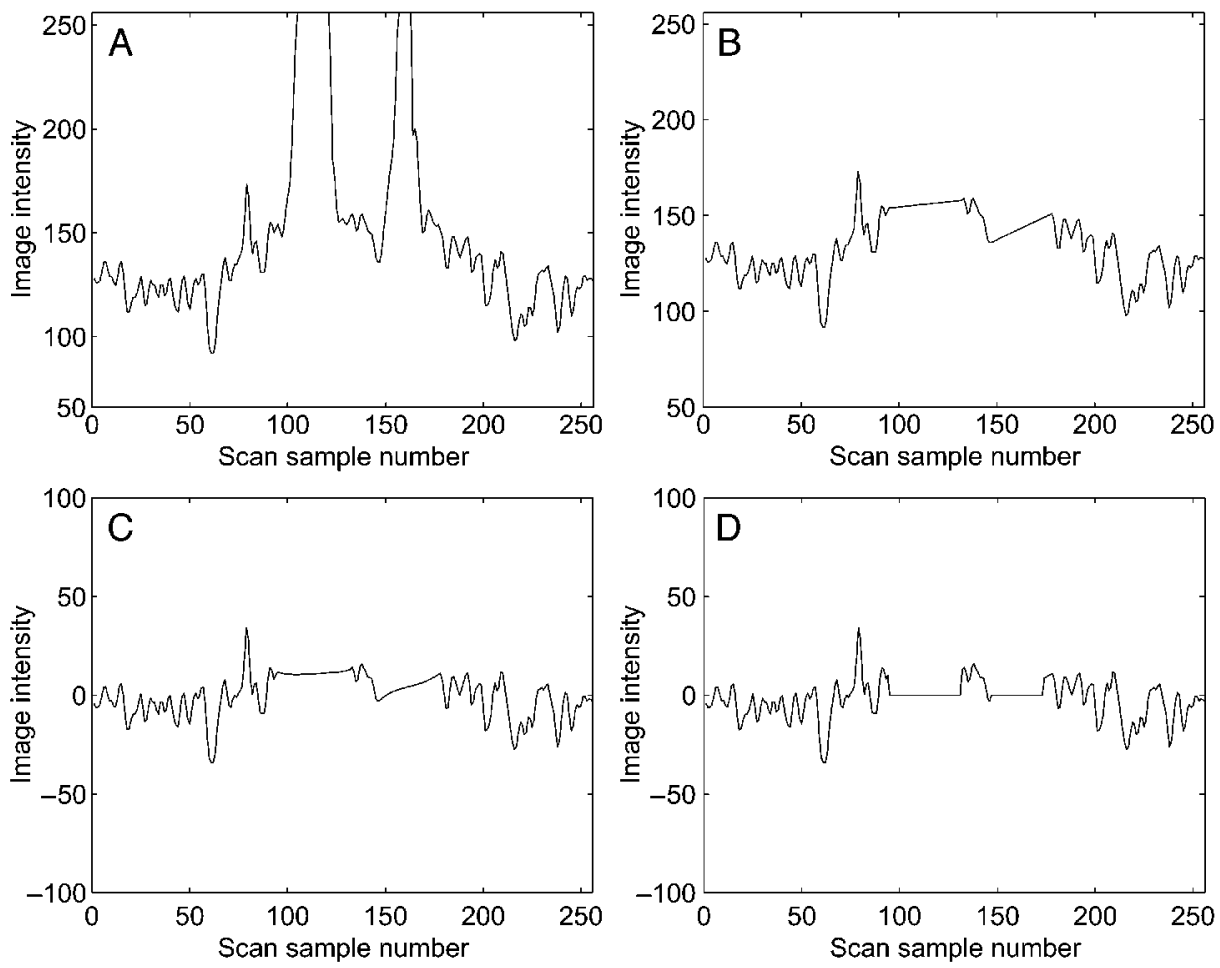

Figure 10. Diagrams illustrating the filtering process to remove intensity gradients on the torsional recordings. (A) Raw torsional recording including two specular reflections. (B) The torsional recording with linear interpolation over areas of specular reflection. (C) The signal shown in panel B filtered with a 32-tap Hamming filter $(\omega=0.15)$. (D) The filtered signal with the original areas of specular reflection set to 0 .

affected by the boundary effects generated by specular reflections or removal of specular reflection by setting values to zero. Prior to filtering, saturating specular reflection is cut out and replaced with data linearly interpolated from the points either side of the areas of specular reflection. The linear interpolation maintains continuity and, therefore, reduces the boundary effects of discontinuous data when the signal is filtered. Figure 10 illustrates the filtering process used to remove intensity gradients from eye tracker torsional data.

\section{EYE-TRACKER PERFORMANCE}

The performance of the laser-based eye tracker has been measured both on artificial eye movement simulators and with human subjects.

\section{Eye Movement Simulators}

The eye tracker's performance in both translational and torsional tracking has been quantified using two prototype artificial eye movement simulators able to provide repeatable and controllable eye movements. The first simulator generated translational movements over a $25^{\circ}$ (radial) range and was capable of oscillating the eye at $10 \mathrm{~Hz}$. It consisted of a prosthetic eye with high-speed servomotors controlling the horizontal and vertical eye movements, as is shown in Figure 11.

The second simulator generated high-speed translational and torsional movements and was constructed of a rotating plate with a positionable prosthetic iris and pupil. The simulator was capable of $47-\mathrm{Hz}$ horizontal, vertical, and torsional movements. The simulators were used to determine the eye trackers maximum eye tracking velocity, bandwidth, resolution, noise, and range. The eye tracker was able to track translational movements of up to $\pm 60^{\circ}$ at velocities of $900 \% \mathrm{sec}$ and torsional movements of up to $\pm 88^{\circ}$ at velocities of $850 \% \mathrm{sec}$. The performance characteristics of the eye tracker are shown in Table 1.

The torsion estimation algorithm is reliant upon accurate positioning of the torsional sweep when recording the iris pattern. During translational eye movement, the scan pattern tracks the pupil as it moves, but the positioning of the torsional sweep becomes progressively offset as translational velocity increases. This affects the torsion estimation cross-correlation algorithm and results in an offset in the torsion estimation. With the current scan rate of 169 scans per second (the maximum rate of real-time computation with the current Pentium 100 PC), torsion estimation is accurate for translational eye movements of less than $70 \% \mathrm{sec}$. This could be improved by rearranging the eye 

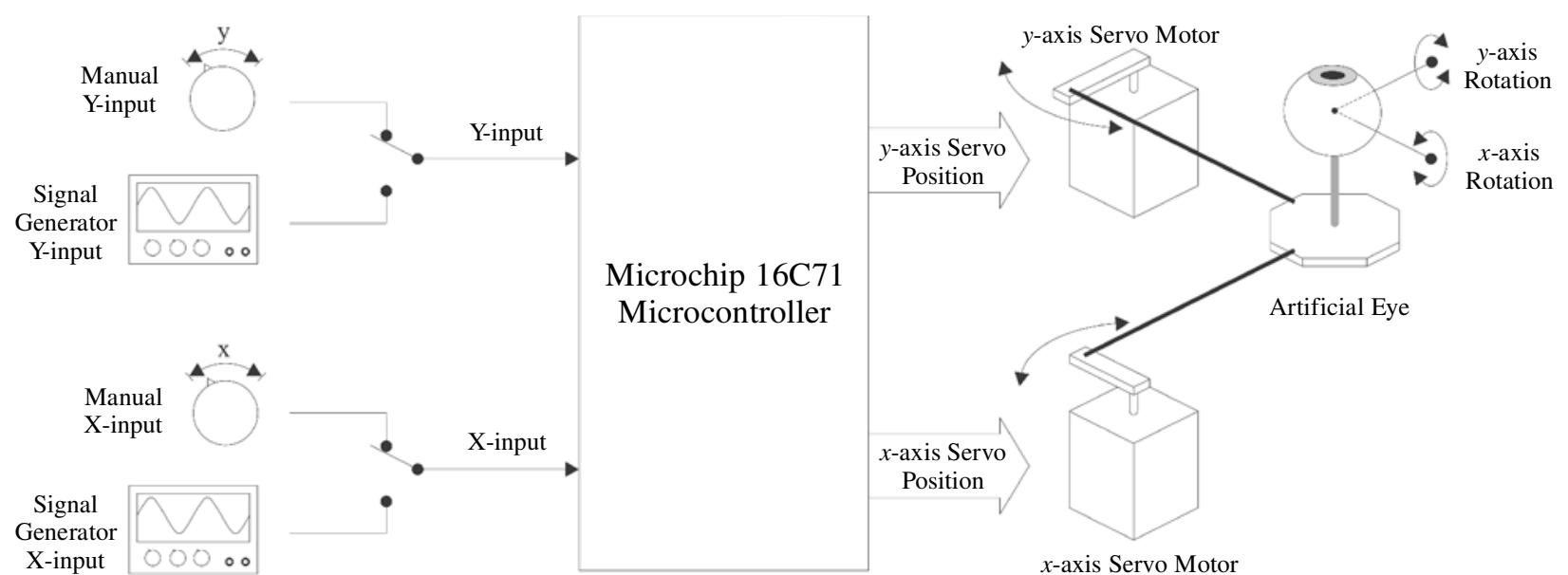

Figure 11. Block diagram of the translational eye movement simulator.

track scan pattern so that the torsional sweep is performed first (to minimize the time for eye movement between the pupil position detection and the torsional sweep) or by increasing the scan rate by increasing computing power. The recording is from the translational eye movement simulator performing $50^{\circ}$ saccades at a velocity of approximately $140 \% \mathrm{sec}$.

\section{Normal Subjects}

Eight normal subjects were tested to evaluate the eye tracker's tracking ability, range, and noise. The test group consisted of 4 females and 4 males, with an average age of 30 years (range, 18-60 years) and a wide range of eye colors. A flat board $1.2 \mathrm{~m}$ square containing a set of eight fixed viewing points (positioned $20^{\circ}$ from central fixa- tion) was used to guide the subjects' eyes in different patterns of up to $\pm 20^{\circ}$ to generate translational movements. The subjects tilted their heads from side to side (i.e., rotation of the head about the naso-occipital axis) to generate torsional ocular counter-roll. Data without large glitches or loss of tracking was obtained from 5 subjects when eye movements were limited to $\pm 14^{\circ}$ horizontally and $\pm 14^{\circ}$ vertically (in 2 of these 5 subjects, $\pm 25^{\circ}$ horizontally and vertically were achieved). Translational performance limitations arise from obstruction of the laser beam by eyelids and eyelashes and, hence, varied from person to person. Enhancements to the mirror/sensor part of the system should be able to improve the range of eye movements for the majority of subjects. Figure 12 shows a torsional recording of ocular counter-roll from a normal subject

Table 1

Performance Summary of the Eye Tracker

\begin{tabular}{|c|c|}
\hline \multicolumn{2}{|r|}{ Translational Performance } \\
\hline Tracking speed: & $900 \% \mathrm{sec}(3-\mathrm{mm}$ pupil diameter) \\
\hline Range (simulators): & $\pm 60^{\circ}$ radius \\
\hline Range (humans): & at least $\pm 14^{\circ}$ horizontal/vertical $\left(20^{\circ}\right.$ oblique $)$ \\
\hline Resolution: & $0.17^{\circ}$ of arc (limit of 8-bit sampling resolution) \\
\hline Bandwidth (3 dB): & $\begin{array}{l}\text { horizontal, }>47 \mathrm{~Hz} \\
\text { vertical, } 30 \mathrm{~Hz}\end{array}$ \\
\hline Noise (stationary simulators): & $\begin{array}{l}\text { horizontal, } 0.13^{\circ} \operatorname{RMS}\left(8^{\prime} \text { of arc }\right) \\
\text { vertical, } 0.12^{\circ} \operatorname{RMS}\left(7^{\prime} \text { of arc }\right)\end{array}$ \\
\hline $\begin{array}{l}\text { Noise (humans focusing } \\
\text { on stationary point object): }\end{array}$ & $\begin{array}{l}\text { horizontal, } 0.12^{\circ} \text { RMS ( } 7^{\prime} \text { of arc) } \\
\text { vertical, } 0.13^{\circ} \text { RMS ( } 8^{\prime} \text { of arc) }\end{array}$ \\
\hline \multicolumn{2}{|r|}{ Torsional Performance } \\
\hline Tracking speed: & $850 \% \mathrm{sec}$ \\
\hline Range: & minimum $\pm 30^{\circ}$, tested on simulators on movements up to $88^{\circ}$ \\
\hline Resolution: & $1.05^{\circ}$ without interpolation \\
\hline Bandwidth: & $\begin{array}{l}\text { unable to test with current simulators; expected to match or better the } \\
\text { translational bandwidth }(>47 \mathrm{~Hz}) \text {; posttorsional filter cutoff set to } 40 \mathrm{~Hz} \text {, for } \\
\text { removal of high-frequency noise }\end{array}$ \\
\hline Noise: & $0.4^{\circ} \mathrm{RMS}$ \\
\hline Reliability: & $\begin{array}{l}\text { torsion estimation valid for translation velocities }<70^{\circ} / \mathrm{sec} \text {. Tracking offsets at this } \\
\text { speed can generate a maximum false torsion equal to the torsional noise level } \\
\text { of } 0.4^{\circ} \text {, which increases with higher speeds; geometrical offsets can cause } \\
\text { false torsion up to } 5^{\circ} \text { at a gaze direction of } 20^{\circ} \text { from center. }\end{array}$ \\
\hline
\end{tabular}



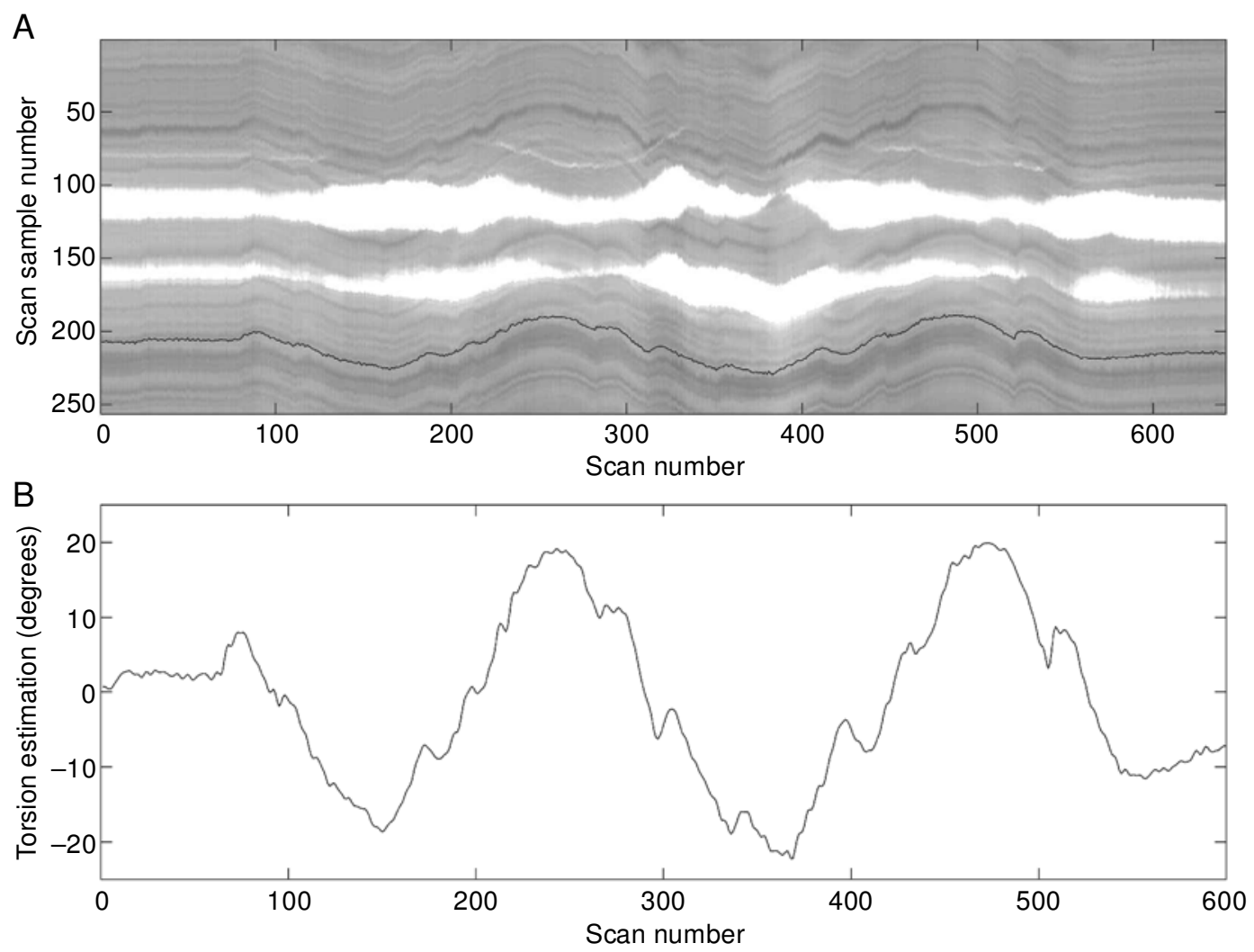

Figure 12. Torsion estimation from a normal subject undergoing ocular counter-roll (vigorous head movement; 600 scans $=3.5 \mathrm{sec}$ of recording). Note the torsional nystagmus (fast and slow phases) during the primary torsional movements. (A) Estimated torsion eye movement overlaid on top of raw torsional data (white data represents areas of saturating specular reflection). (B) A larger view of the torsion estimation trace.

vigorously tilting his head from side to side (approximately $3.5 \mathrm{sec}$ ). The maximum torsional movements observed and recorded were $30^{\circ}$.

Figures $13 \mathrm{~A}$ and $13 \mathrm{~B}$ show recordings of subjects performing saccades. The fidelity of the high-frequency components of the saccades can be seen to be most acceptable.

Poor test data (data containing large glitches) were obtained from 2 subjects, owing to mechanical constraints (deep-set eyes, nose shape, and nose size) that prevented the eye tracker optics (laser, mirrors, and sensors) from being centered optimally over the subject's pupil (translational offsets of the optics from the center of the pupil were equivalent to $37^{\circ}$ and $14^{\circ}$ ). The tests were still performed, but the results were noisy and often showed total loss of tracking. No useful data (owing to failure of tracking) could be obtained from 1 of the subjects because of the very dark iris coloring (difficult to visually differentiate pupil from iris).

\section{Clinical Cases}

Eye movement recordings were recorded in a patient who had suffered a recent cerebellar stroke. Several pathological abnormalities were evident in both the horizontal and the vertical eye movement recordings (Figure 13B), including ocular dysmetria (overshooting when saccadic movements were made), gaze-evoked horizontal nystagmus, and a high number of square-wave jerks. The latter can also be seen in normal subjects but occur much less frequently. No abnormal torsional movements were observed.

We have also used the device to record torsional pulsion of saccades in patients with neurological disorders (Anderson \& MacAskill, 1998).

\section{CONCLUSION}

A noninvasive laser-based eye tracker has been developed that can measure high-speed eye movements in all three axes of eye rotation. On a set of 8 normal subjects, the translational tracking was found to be reliable and accurate on 5 subjects over a range of at least $\pm 14^{\circ}$ horizontally and vertically. It is expected that the performance of 2 of the remaining subjects would be at the same level following improvements to the eye tracker headset to allow for better positioning of the eye tracker optics on the eye. Currently, the eye tracker can record eye movement only in one eye at a time and is unable to measure eye movements in people with extremely dark or absent irises.

Overall, the eye tracker compares well with other noninvasive eye-tracking systems and provides an alternative, 

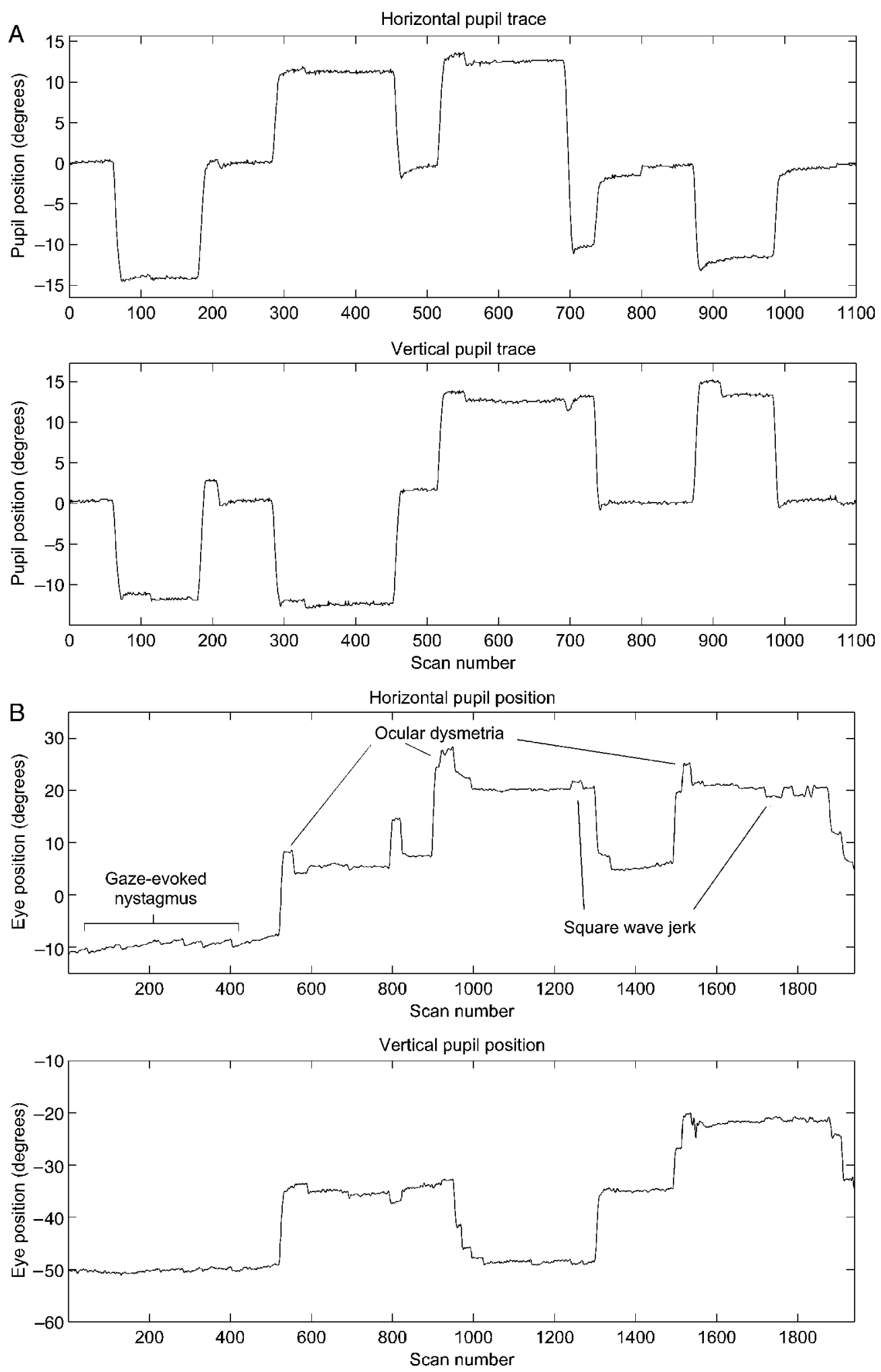

Figure 13. Translational eye movement recordings from the eye tracker. (A) Horizontal and vertical traces from a normal subject performing oblique (diagonal) saccades $(1,100 \mathrm{scans}=6.5 \mathrm{sec}$ of recording). Note the mild disconjugacy of the eyes during horizontal saccades. (B) Vertical and horizontal eye movements in a patient who had suffered a cerebellar stroke $(1,940 \mathrm{scans}=11.5 \mathrm{sec}$ of recording $)$. 
novel method for noninvasive simultaneous measurement of translational and torsional eye movements.

\section{REFERENCES}

Anderson, T. J., \& MacAskill, M. R. (1998). Pathological torsional pulsion of saccades: An observational study in seven patients. New Zealand Medical Journal, 111, 480-481.

Clarke, A. H., Ditterich, J., Drüen, K., Schönfeld, U., \& STEINEKE, C. (2002). Using high frame rate CMOS sensors for threedimensional eye tracking. Behavior Research Methods, Instruments, \& Computers, 34, 549-560.

ECKERT, R. (1996). Laser scanning eye tracker (Tech. Rep.). Christchurch, New Zealand: University of Canterbury, Department of Electrical and Electronic Engineering.

Jones, R. D., Wilson B. A., \& Bones, P. J. (1996). A flying-spot laser scanner for tracking eye movements. In Proceedings of the IEEE 8th Annual International Conference of the Engineering in Medicine and Biology Society (pp.146-147). Los Alamitos, CA: IEEE Computer Society Press.

KaWAi, H., Tamura, S., Kani, K., \& KariYa, K. (1986). Eye movement analysis system using fundus images. Pattern Recognition, 19, 77-84.

LEIGH, R. J., \& ZEE, D. S. (1999). The neurology of eye movements (3rd ed.). New York: Oxford University Press.

Matin, L., \& Pearce, D. (1964). Three dimensional recording of rota- tional eye movements by a new contact lens technique. Biomedical \& Scientific Instrumentation, 2, 79-95.

Mulligan, J. B. (1997). Image processing for improved eye-tracking accuracy. Behavior Research Methods, Instruments, \& Computers, 29, 54-65.

Robinson, B. (1963). A method of measuring eye movement using a scleral search coil in a magnetic field. IEEE Transactions on Biomedical Engineering, 10, 137-145.

Wilson, B. A., Anderson T., Preddie J., Jones, R. D., \& Bones, P. J. (1996a). A flying spot laser scanner capable of measuring torsional eyemovements [Abstract]. Journal of Vestibular Research, 6 (Suppl.), S75.

Wilson, B. A., Anderson T., Preddie J., Jones, R. D., \& Bones, P. J. (1996b). A flying spot laser scanner capable of measuring torsional eyemovements [Abstract]. Neuro-Opthalmology, 16 (Suppl.), 177.

Wilson, B. A., Jones, R. D., \& Bones, P. J. (1995). A flying spot laser scanner for tracking eye-movements [Abstract]. New Zealand Medical Journal, 108, 487.

Young, L. R., \& Sheena, D. (1975). Survey of eye movement recording methods. Behavior Research Methods \& Instrumentation, 7, $397-$ 429.

(Manuscript received March 25, 2002; revision accepted for publication October 14, 2002.) 